\title{
SPECTROSCOPY OF SPATIALLY EXTENDED MATERIAL AROUND HIGH-REDSHIFT RADIO-LOUD QUASARS
}

\author{
Timothy M. Heckman, ${ }^{1,2,3}$ Matthew D. Lehnert, ${ }^{1,2,3}$ George K. Miley, ${ }^{2,4}$ ANd Wil VAN Breugel ${ }^{5}$ \\ Received 1991 March 18; accepted 1991 May 15
}

\begin{abstract}
We present the analysis of long-slit optical spectra taken of five high-redshift $(z \approx 2-3)$ radio-loud quasars (QSRs), selected from the imaging survey of Heckman et al. We confirm that the QSRs have Ly $\alpha$ emission that is spatially extended by at least several tens of kiloparsecs, with a luminosity of order $10^{44} \mathrm{ergs} \mathrm{s}^{-1}$. The gas is very active kinematically. After a subtraction of the seeing-scattered light from the broad-line region (BLR) of the central QSR, we find that line widths in the nebulae are $1000-1500 \mathrm{~km} \mathrm{~s}^{-1}$ (FWHM). The gas does not show any globally organized velocity gradients (i.e., velocities do not change by more than about 500 $\mathrm{km} \mathrm{s}^{-1}$ over scales of tens of kiloparsecs). The line widths are close to the maximum possible for gas that is freely falling into the potential well of a very massive galaxy. Alternatively, we may be witnessing the explosive ejection of matter on a galactic scale by the QSR. The kinematic properties of the QSR nebulae are broadly similar to those of the nebulae associated with high-redshift radio galaxies.

We confirm the suggestion of Foltz et al. that the nuclear He II $\lambda 1640$ emission line in QSRs is systematically narrower than other nuclear emission lines. We find that this narrow-lined, nuclear He II emission has a strength that correlates with the strength of the spatially extended Ly $\alpha$ emission, and suggest that the former probably arises in the inner (arcsec-scale) part of the Ly $\alpha$ nebula. This is consistent with the fact that the velocity of the spatially extended Ly $\alpha$ emission agrees well with the velocity of the narrow core of the nuclear Ly $\alpha$ profile. Selecting by the presence of strong narrow He II $\lambda 1640$ lines should be a good way to find high- $z$ quasars with prominent Ly $\alpha$ nebulae. We discuss the implications of these results for the nature of the $z_{\mathrm{abs}} \approx$ $z_{\text {em }}$ systems in high- $z$ QSRs. In three QSRs, the spatially extended Ly $\alpha$ is redshifted by of order $10^{3} \mathrm{~km} \mathrm{~s}^{-1}$ with respect to the C IV $\lambda 1549$ BLR profile (but exhibits no significant shift in the other two QSRs). We believe that this reflects a blueshift of the $C$ IV lines with respect to QSR systemic velocity. This has implications for the interpretation of the BLR dynamics.

We have likely $(4-5 \sigma)$ detections of spatially extended He II $\lambda 1640$ in one QSR nebulae and C IV $\lambda 1549$ in another (each at $7 \%-10 \%$ of $\mathrm{Ly} \alpha$ ). These possible detections and the upper limits on other lines are consistent with the emission-line spectra of high- $z$ radio galaxies. Our data are not good enough either to yield strong constraints on the metallicities in the nebulae or to discriminate decisively between alternative ionization sources (photoionization by a standard active galactic nucleus continuum, photoionization by hot stars, shock heating, etc.). The probable He II detector is inconsistent with predictions of photoionization models for normal high-mass stars. The probable $\mathrm{C}$ IV detection means that at least some galaxy-scale sites in the early universe have nonprimordial chemical abundances. The probable detections of $\mathrm{He}$ II and $\mathrm{C}$ IV make it unlikely that the bulk of the spatially extended Ly $\alpha$ emission arises via resonant scattering of nuclear Ly $\alpha$ photons by neutral hydrogen surrounding the QSR.
\end{abstract}

Subject headings: galaxies: formation - galaxies: internal motions - quasars

\section{INTRODUCTION}

The recent discovery by Heckman et al. (1991, hereafter HLVM) of large $(\approx 100 \mathrm{kpc})$, luminous $\left(\approx 10^{44} \mathrm{ergs} \mathrm{s}^{-1}\right)$ Ly $\alpha$ nebulae around 15 radio-loud quasars (QSRs) at $z \approx 2-3$, and the possibly related phenomena discussed recently by Djorgovski et al. (1987b), Hu et al. (1991), and Steidel, Sargent, \& Dickinson (1991) offer us the opportunity to investigate a number of important issues from a fresh perspective.

\footnotetext{
${ }^{1}$ Rowland Department of Physics and Astronomy, Johns Hopkins University, Baltimore, MD 21218.

${ }^{2}$ Space Telescope Science Institute, 3700 San Martin Drive, Baltimore, MD 21218.

${ }^{3}$ Visiting Observer at the Kitt Peak National Observatory of the National Optical Astronomy Observatories, operated by AURA under contract with the National Science Foundation.

${ }^{4}$ Sterrewacht Leiden, Postbus 9513, 2300RA, Leiden, The Netherlands.

5 Institute for Geophysics and Planetary Physics, Lawrence Livermore National Laboratories, L-413, Livermore, CA 94550.
}

First, quite general arguments imply that significant infall into galaxies must have been occurring at redshifts of several (Rees 1988). Indeed, hierarchical-clustering cosmogonical models (e.g., Carlberg \& Couchman 1989) predict that galaxy formation occurred relatively late $(z \approx a$ few $)$. By studying the environments of high-z QSRs we may therefore be able to observe the processes of galaxy formation at work. A specific "galaxy formation" model has been advocated by Fabian and coworkers in the context of QSRs at redshifts of $\approx 1$ (cf. Crawford \& Fabian 1989; Forbes et al. 1990): QSRs are immersed in "cooling flows" with cooling/infall rates that are so large that we are in essence observing the formation of a massive galaxy. A comparison of QSR nebulae to those associated with low $-z$ cooling flows (Heckman et al. 1989; Hu 1988) should be particularly instructive.

Second, the comparative study of the gaseous environments of QSRs at high and low redshifts (Stockton \& MacKenty 1987; Boroson, Persson, \& Oke 1985; Forbes et al. 1990; HLVM) may help explain how and why the QSR population 
has evolved so strongly with cosmic time and how conditions in the early universe may have been different. These studies may also help explain the observed redshift dependence of the basic properties (size, morphology, amount of depolarization) of the nonthermal QSR radio sources (Miley 1971; Barthel \& Miley 1988; Garrington \& Conway 1991; Neff \& Hutchings 1990).

Third, Ikeuchi (1981) has suggested that quasars were important sources of mechanical energy in the early universe and regulated the thermal and dynamical properties of the intergalactic medium (see also Ikeuchi \& Ostriker 1986). Can we directly observe the outflow of mechanical energy in the Ly $\alpha$ nebulae around high- $z$ QSRs?

Fourth, various "unification schemes" for active galactic nuclei (AGNs) maintain that QSRs and radio galaxies are related through either viewing angle, time-dependent, or environmental factors (e.g., Barthel 1989; Norman \& Miley 1984). Observations of the properties of the spatially resolved material in the two types of objects can help test these hypotheses.

In this paper, we present the results of spectroscopy of five QSRs selected from the HLVM imaging survey to have bright, spatially extended Ly $\alpha$ emission. While the images provide basic information about the size, morphology, and luminosity of the nebulae, they do not allow us to understand the physical or dynamical state of the gas. We therefore undertook this spectroscopic program to address the following specific questions: Is there any kinematic evidence for outflow, infall, or rotation? Are the relative line intensities in the nebulae consistent with photoionization by the QSR or massive stars? How do the kinematics and physical conditions in the QSR nebulae compare to those of high- $z$ radio galaxy nebulae? How do the properties of the nebulae compare to those of nebulae associated with low-redshift "cooling flows"? Are the properties of the nebulae consistent with the properties of the population of absorbing clouds seen near the QSR emission-line redshift (the " $z_{\text {abs }} \approx z_{\text {em }}$ systems"; see Anderson et al. 1987; Foltz et al. 1988)?

\section{OBSERVATIONS AND DATA REDUCTION}

The objects we observed were all QSRs with radio structures having scales $\geq 1^{\prime \prime}$ ( $\geq 10 \mathrm{kpc}$ ). These QSRs were drawn from a much larger sample whose radio and optical properties are discussed extensively elsewhere (HLVM; Barthel et al. 1988; Barthel, Tytler, \& Thompson 1990, hereafter BTT). The specific QSRs we observed were selected to have bright, spatially resolved Ly $\alpha$ emission.

Our spectra were obtained with the R-C spectrograph (with a UV-flooded TI CCD detector) on the KPNO $4 \mathrm{~m}$ Mayall telescope on the nights of 1990 January 27 and 28 . We used the BL 250 grating (300 lines per $\mathrm{mm}$ ) in first order. For four of the QSRs we used a 1".5 slit width and employed the CCD in its full $800 \times 800$ mode, yielding pixel sizes of 0 ".43 by $3.8 \AA$ and a spectral resolution of $10.5 \AA$ FWHM. We observed $\mathrm{Q} 0941+261$ with a $2^{\prime \prime}$ slit width and $2 \times 2$ pixel binning $(400 \times 400$ mode), yielding pixel sizes 0.86 by $7.6 \AA$ and a spectral resolution of $14.5 \AA$. The free spectral range in all these cases was about $3000 \AA$, allowing us to cover the wavelength range from about 1100 to $1900 \AA$ in the QSR rest frame. Integration times were $9000 \mathrm{~s}$ for $\mathrm{Q} 0445+097,8100 \mathrm{~s}$ for $\mathrm{Q} 0758+120,5000 \mathrm{~s}$ for $\mathrm{Q} 1318+113$, and $3600 \mathrm{~s}$ for Q0805 + 046 and Q0941 + 261. All spectra were taken centered on the QSR proper and oriented along the position angle with the brightest $\mathrm{Ly} \alpha$ emission (as judged from the images in HLVM). Conditions were photometric, and the seeing was $1^{\prime \prime} 0$ to 1.5 FWHM (except for Q0941 + 261 where the seeing was $\approx 2^{\prime \prime}$ ).

Reduction of the spectra was done using the "Longslit" package in IRAF. After subtracting the bias using the overscan columns, the spectra were flat-fielded using exposures taken of an internal quartz lamp. Wavelength calibration and geometric rectification of the spectra were done using spectra of He-Ne-Ar comparison lamps which were observed immediately before and after each QSR. The sky contribution was then subtracted interactively using the task "Background" in IRAF. We used observations of the standard star HZ 44 obtained through a 4 " wide slit to flux calibrate three of the spectra. We did not observe a standard star for Q0758+120 and Q0805+046. We have, however, placed the Ly $\alpha$ line emission in these two spectra on a flux scale by using the fluxcalibrated broad-band ( $U$ or $B$ ) images in HLVM to calibrate the QSR continuum in our spectra and then determining Ly $\alpha$ fluxes from our spectroscopically measured Ly $\alpha$ equivalent widths.

Since we were interested in determining the properties of the intrinsic emission of each QSR nebula, it was first necessary to subtract the contribution of light from the central (unresolved) QSR that had been "scattered" into the nebula by the instrumental point-spread function. We did this by first forming a spectrum of the QSR using a sum of the central-most region $\approx 1$.5 in size centered on the QSR continuum peak. We then examined the "off-nuclear" columns on each side of the QSR and iteratively subtracted a scaled version of the spectrum of the central QSR so that all the continuum near Ly $\alpha$ and all traces of the very broad $\left(\approx 10^{4} \mathrm{~km}^{-1} \mathrm{FWHM}\right)$ Ly $\alpha$ emission lines from the broad line region (BLR) vanished. Note that this procedure explicitly assumes that the BLR and UV continuum is spatially unresolved. This is a deliberately conservative assumption that minimizes the amount of nebular line emission left in our seeing-corrected off-nuclear spectra. In general, we found that it was necessary to substract significant amounts of scattered nuclear light only in the columns within $\approx 2$ " of the QSR.

Because the position angles observed were dictated by the nebular structure, we were not able to minimize effectively the component of differential atmospheric refraction across the slit. Using the formalism and tables in Filippenko (1982), we find that differential refraction perpendicular to our 1 ".5 or $2^{\prime \prime}$ wide slits was negligible $\left(\leq 0^{\prime \prime} 25\right)$ except for the spectrum of $\mathrm{Q} 0805+046$ where it amounts to $\approx 1^{\prime \prime}$ between the blue and red ends of the spectrum. As a further check on this effect, we obtained short-exposure spectra of each QSR with a 4" slit, and estimated the amount of light refracted out of the slit by comparing these spectra to spectra obtained with the 1.5 slit. The continuum shapes in the wide and narrow slits agree to better than $10 \%$ except for the spectrum of Q0805 + 046 where the shape differed by $30 \%$. Since this spectrum was not fluxcalibrated anyway, we have not corrected it for differential refraction. We expect the effects of differential refraction on the measured nebular Ly $\alpha$ parameters to be minimal anyway, since the correction for seeing-scattered nuclear light was made using the portion of the nuclear spectrum that was at or very near the wavelength of $L y \alpha$ (see above).

\section{RESULTS}

We display the spectra of the QSR nuclei in Figure 1 and the spectra of the nebulae (summed over all the spatial increments 

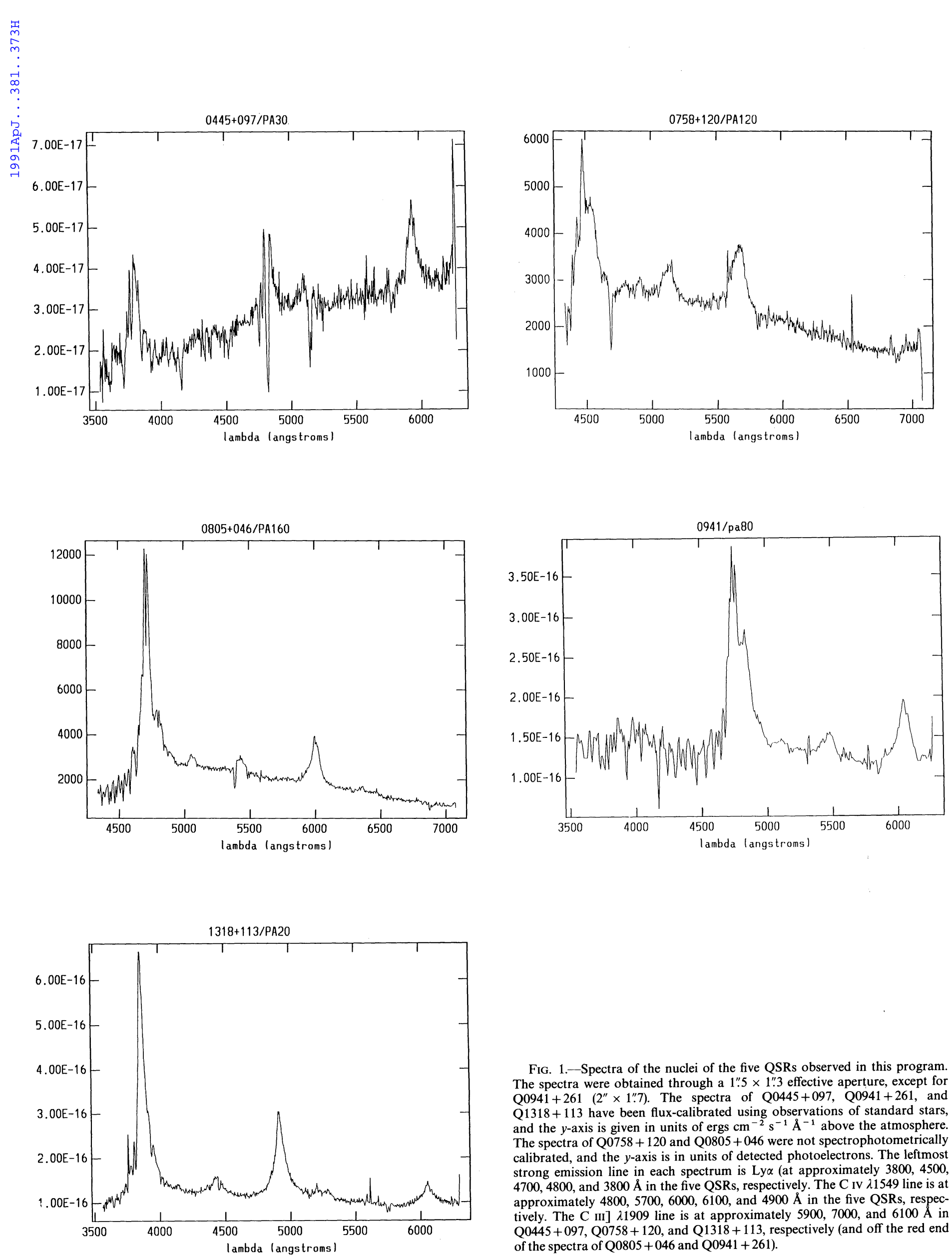

FIG. 1.- Spectra of the nuclei of the five QSRs observed in this program. The spectra were obtained through a 1".5 $\times 1$ 1.3 effective aperture, except for $\mathrm{Q} 0941+261\left(2^{\prime \prime} \times 11^{\prime \prime} 7\right)$. The spectra of Q0445+097, Q0941+261, and Q1318+113 have been flux-calibrated using observations of standard stars, and the $y$-axis is given in units of ergs $\mathrm{cm}^{-2} \mathrm{~s}^{-1} \AA^{-1}$ above the atmosphere. The spectra of Q0758 + 120 and Q $0805+046$ were not spectrophotometrically calibrated, and the $y$-axis is in units of detected photoelectrons. The leftmost strong emission line in each spectrum is Ly $\alpha$ (at approximately 3800,4500 , 4700,4800 , and $3800 \AA$ in the five QSRs, respectively. The $C$ IV $\lambda 1549$ line is at approximately $4800,5700,6000,6100$, and $4900 \AA$ in the five QSRs, respectively. The $C$ III] $\lambda 1909$ line is at approximately 5900,7000 , and $6100 \AA$ in $\mathrm{Q} 0445+097, \mathrm{Q} 0758+120$, and Q1318 + 113, respectively (and off the red end of the spectra of Q0805+ 046 and Q0941 + 261). 

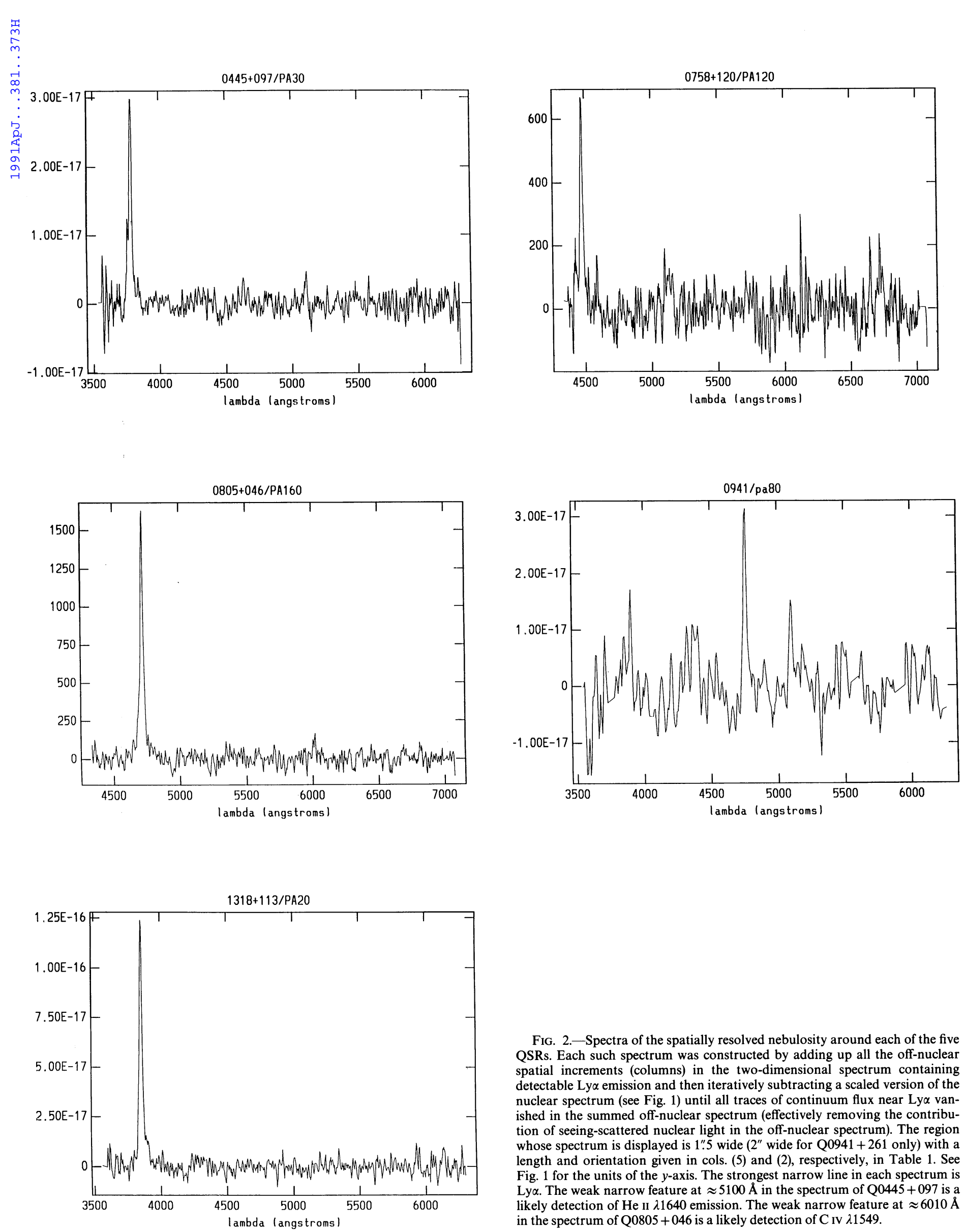

FIG. 2.-Spectra of the spatially resolved nebulosity around each of the five QSRs. Each such spectrum was constructed by adding up all the off-nuclear spatial increments (columns) in the two-dimensional spectrum containing detectable Ly $\alpha$ emission and then iteratively subtracting a scaled version of the nuclear spectrum (see Fig. 1) until all traces of continuum flux near Ly $\alpha$ vanished in the summed off-nuclear spectrum (effectively removing the contribution of seeing-scattered nuclear light in the off-nuclear spectrum). The region whose spectrum is displayed is $1^{\prime \prime} .5$ wide $\left(2^{\prime \prime}\right.$ wide for Q0941 + 261 only) with a length and orientation given in cols. (5) and (2), respectively, in Table 1. See Fig. 1 for the units of the $y$-axis. The strongest narrow line in each spectrum is Ly $\alpha$. The weak narrow feature at $\approx 5100 \AA$ in the spectrum of $Q 0445+097$ is a likely detection of $\mathrm{He}$ II $\lambda 1640$ emission. The weak narrow feature at $\approx 6010 \AA$ in the spectrum of Q0805+ 046 is a likely detection of C IV $\lambda 1549$. 

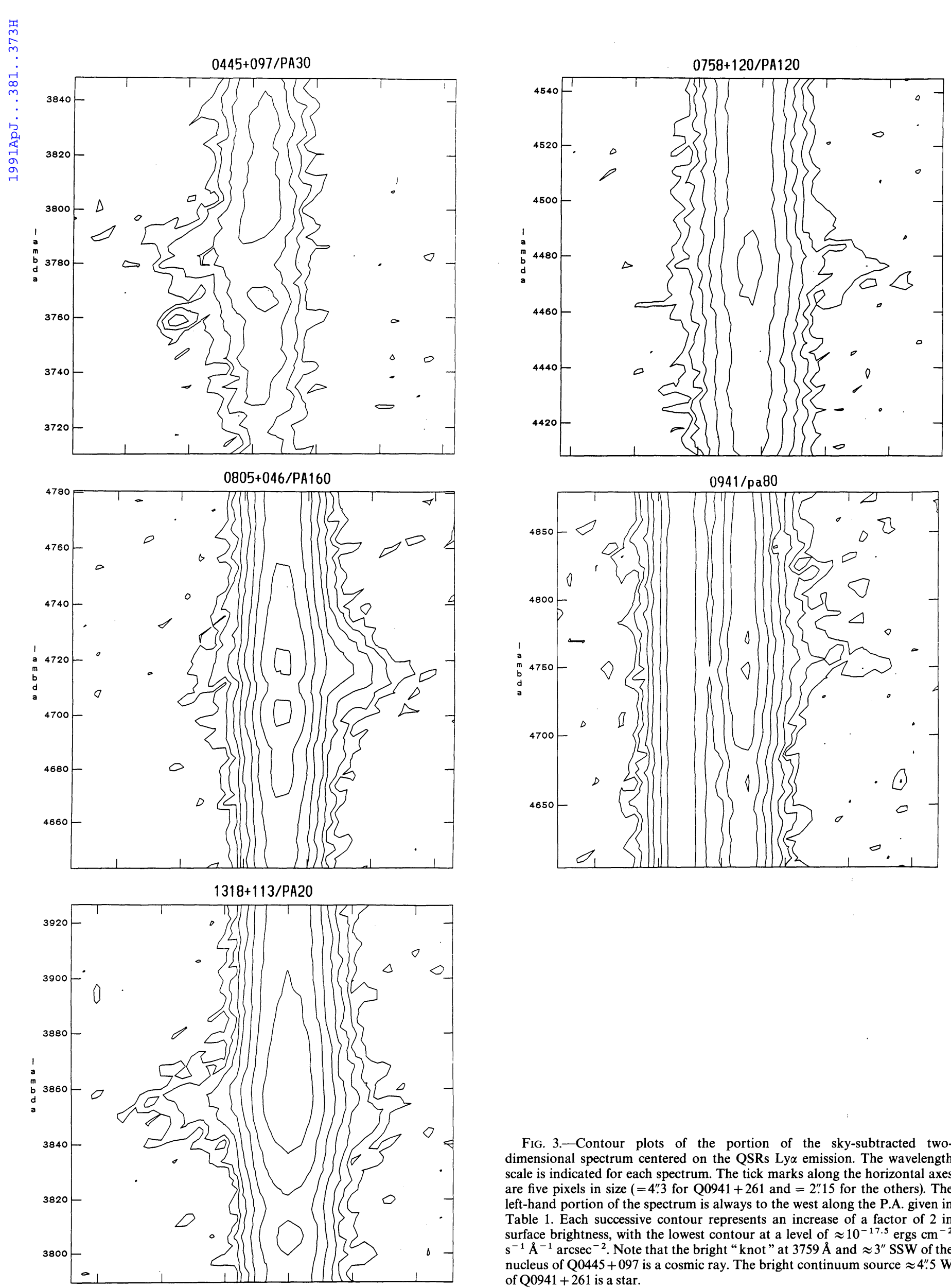

FIG. 3.-Contour plots of the portion of the sky-subtracted twodimensional spectrum centered on the QSRs Ly $\alpha$ emission. The wavelength scale is indicated for each spectrum. The tick marks along the horizontal axes are five pixels in size (=4"3 for Q0941 + 261 and = 2".15 for the others). The left-hand portion of the spectrum is always to the west along the P.A. given in Table 1. Each successive contour represents an increase of a factor of 2 in surface brightness, with the lowest contour at a level of $\approx 10^{-17.5} \mathrm{ergs} \mathrm{cm}^{-2}$ $\mathrm{s}^{-1} \AA^{-1} \operatorname{arcsec}^{-2}$. Note that the bright "knot " at $3759 \AA$ and $\approx 3^{\prime \prime}$ SSW of the nucleus of $\mathrm{Q} 0445+097$ is a cosmic ray. The bright continuum source $\approx 4$ ".5 W of Q0941 + 261 is a star. 
TABLE 1

Nebular Structural Properties

\begin{tabular}{|c|c|c|c|c|c|c|c|}
\hline $\begin{array}{l}\text { QSR } \\
\text { (1) }\end{array}$ & $\begin{array}{l}\text { P.A. } \\
\text { (2) }\end{array}$ & $\underset{\text { (3) }}{\log F_{s}}$ & $\underset{\text { (4) }}{\log F_{i}}$ & $\begin{array}{l}D_{s} \\
(5)\end{array}$ & $\begin{array}{l}D_{i} \\
(6)\end{array}$ & $\begin{array}{l}r_{0} \\
\text { (7) }\end{array}$ & $\begin{array}{c}\text { Scale } \\
(8)\end{array}$ \\
\hline $\mathrm{Q} 0445+097$. & 30 & -15.1 & -14.7 & 4 & 12 & 2.4 & 8.4 \\
\hline $\mathrm{Q} 0758+120$ & 120 & -15.4 & -14.8 & 5 & 13 & 2.0 & 9.2 \\
\hline $\mathrm{Q} 0805+046$. & 160 & -14.6 & -14.1 & 8 & 14 & 1.6 & 9.3 \\
\hline $\mathrm{Q} 0941+261 .$. & 80 & -15.2 & -15.1 & 10 & 12 & 3.2 & 9.3 \\
\hline $\mathrm{Q} 1318+113 \ldots$ & 20 & -14.6 & -14.2 & 9 & 11 & 1.5 & 8.6 \\
\hline
\end{tabular}

NoTES. - (2) The position angle along which the spectrum was taken (in degrees east of north). (3) The log of the spatially resolved Ly $\alpha$ flux (ergs $\mathrm{cm}^{-2}$ $\mathrm{s}^{-1}$ ) from the nebulae, as measured along the slit position angle given in col. (2). These are uncertain by $\approx 0.2$ dex due to photometric uncertainties associated with our 1".5 wide slit and due to the difficulty in properly subtracting off the seeing-scattered nuclear light. (4) The log of the spatially resolved Ly $\alpha$ flux from the nebulae, as measured from the narrow-band images discussed by HLVM. The uncertainties are $0.1-0.3$ dex (see HLVM for details). These fluxes are typically a factor $\approx 2-3$ larger than the fluxes in col. (3), because our slit spectra do not sample the entire nebula. (5) The maximum angular extent of the detectable Ly $\alpha$ emission (in arcsec) in our spectra at a typical isophote of $\approx 5 \times 10^{-17} \mathrm{ergs} \mathrm{cm}^{-2} \mathrm{~s}^{-1} \operatorname{arcsec}^{-2}$. Emission is detected on both sides of the QSR in Q0805+046 and Q1318 + 113, but only on one side in the others. (6) The maximum angular extent of the detectable Ly $\alpha$ emission in the narrowband images in HLVM. These are larger than the values in col. (5) because the images go deeper (typical limiting isophote at $2 \pm 1 \times 10^{-17} \mathrm{ergs} \mathrm{cm}^{-2} \mathrm{~s}^{-1}$ $\operatorname{arcsec}^{-2}$ ) and because the gas is not always maximally extended along the position angles of the spectra. (7) The characteristic $e$-folding length scales (in arcsec) for the surface brightness profiles of the spatially resolved Ly $\alpha$ emission as measured from our spectra. These are uncertain by a factor of about 0.2 dex. (8) The scale (kpc per arcsec) for cosmological parameters $H_{0}=75 \mathrm{~km} \mathrm{~s}^{-1}$ $\mathrm{Mpc}^{-1}$ and $q_{0}=0$. At these redshifts $(z \approx 2-3)$, the scale is about the same as for an $H_{0}=50, q_{0}=\frac{1}{2}$ cosmology.

with detectable emission) in Figure 2. A contour plot of the portion of the two-dimensional spectrum centered on the QSR Ly $\alpha$ emission is shown for each QSR in Figure 3. The striking differences in the relative strengths and widths of the emission lines in the nuclear and off-nuclear spectra demonstrate that we have been quite successful in removing seeing-scattered nuclear light from the off-nuclear spectra.

Note that in the results to follow, we have adopted an "agnostic's cosmology" of $H_{0}=75 \mathrm{~km} \mathrm{~s}^{-1} \mathrm{Mpc}^{-1}$ and $q_{0}=$ 0 , leading to an approximate scale of $9 \mathrm{kpc} \operatorname{arcsec}^{-1}$ (see Table 1 for details). Taking a "theorist's cosmology" of $H_{0}=50 \mathrm{~km}$ $\mathrm{s}^{-1} \mathrm{Mpc}^{-1}$ and $q_{0}=\frac{1}{2}$ would yield a very similar scale factor for this redshift range.

\subsection{Photometric Structure of the Nebulae}

The principal measured structural properties of the nebular emission are listed in Table 1 . The agreement in the total flux and angular size of the nebulae between our spectra and the images in HLVM is satisfactory, given the partial coverage of the nebular emission by our slit and uncertainties in the absolute flux calibration introduced by our narrow slit. The Lya surface brightness profiles fall with radius and are roughly linear when plotted as log (surface brightness) versus radius. The typical implied $e$-folding length scales are $\approx 20 \mathrm{kpc}$ for the QSR nebulae, and the total sizes of the regions of detectable Ly $\alpha$ emission range from $\approx 30-90 \mathrm{kpc}$ along the chosen position angles.

\subsection{Kinematic Structure of the Nebulae}

The principal kinematic properties of the Ly $\alpha$ nebulae are summarized in Table 2 . The most striking result is that the lines are quite broad. After correction for the $\approx 800 \mathrm{~km} \mathrm{~s}^{-1}$ FWHM spectral resolution of our data, we measure average line widths (FWHM) of $1000-1500 \mathrm{~km} \mathrm{~s}^{-1}$ (similar to the line widths observed in high- $z$ radio galaxies; cf. Chambers, Miley, $\&$ van Breugel 1990; McCarthy et al. 1990). Given the breadth of the lines, it is somewhat surprising that we do not detect any large $\left(>500 \mathrm{~km} \mathrm{~s}^{-1}\right)$ change in radial velocity across any of the nebulae (that is, the large-scale shear is less than the typical line-of-sight velocity dispersion).

We have compared the mean velocity of the off-nuclear Ly $\alpha$ line to the mean velocity of the "narrow" (centralmost few thousand $\mathrm{km} \mathrm{s}^{-1}$ ) core of the nuclear Ly $\alpha$ emission line (see Fig. 1 and Table 2). In all cases but Q0445+097 this nuclear material seems to exhibit kinematic continuity with the extranuclear Ly $\alpha$ emission and may be plausibly interpreted as the innermost (spatially unresolved) portion of the nebula. The nuclear Ly $\alpha$ emission line in Q0445+097 is decimated by strong " $z_{\text {abs }} \approx z_{\text {em }}$ " Ly $\alpha$ and $N$ v $\lambda 1240$ absorption, and no clear " narrow" Ly $\alpha$ emission core can be identified.

We also list in Table 2 the velocity of the spatially extended Ly $\alpha$ relative to the nuclear C IV $\lambda 1549$ BLR profile. This latter line is second in strength only to Ly $\alpha$ in our QSR nuclear spectra and is much less affected by absorption than the nuclear Ly $\alpha$ profile (whose blue side is plagued by the Ly $\alpha$ "forest"). In three cases (Q0758 + 120, Q0805+046, and Q0941 + 261) the C IV BLR profile is significantly blueshifted (by $750 \mathrm{~km} \mathrm{~s}^{-1}$ to nearly $2000 \mathrm{~km} \mathrm{~s}^{-1}$ ) with respect to the

TABLE 2

Kinematic Properties of the Nebulae

\begin{tabular}{|c|c|c|c|c|c|c|}
\hline $\begin{array}{c}\text { QSR } \\
(1)\end{array}$ & $\begin{array}{c}\langle\mathrm{FWHM}\rangle \\
(2)\end{array}$ & $\begin{array}{c}z_{\text {Ly } \alpha, \text { neb }} \\
\text { (3) }\end{array}$ & $\begin{array}{l}z_{\text {Ly } \alpha, \text { nuc }} \\
\text { (4) }\end{array}$ & $\Delta v_{\mathrm{Ly \alpha}}$ & $\begin{array}{c}z_{\mathrm{CIV}} \\
(6)\end{array}$ & $\Delta v_{\text {CIV }}$ \\
\hline $\mathrm{Q} 0445+097 .$. & $1500 \pm 300$ & $2.113 \pm 0.002$ & & & 2.114 & $-100 \pm 300$ \\
\hline $\mathrm{Q} 0758+120 \ldots$ & $1100 \pm 400$ & $2.682 \pm 0.003$ & 2.683 & $-100 \pm 300$ & 2.657 & $2000 \pm 300$ \\
\hline $\mathrm{Q} 0805+046 \ldots$ & $1100 \pm 200$ & $2.880 \pm 0.002$ & 2.877 & $200 \pm 200$ & 2.870 & $800 \pm 200$ \\
\hline Q $0941+261 \ldots \ldots \ldots \ldots$ & $1000 \pm 400$ & $2.914 \pm 0.003$ & 2.913 & $100 \pm 300$ & 2.901 & $1000 \pm 300$ \\
\hline $\mathrm{Q} 1318+113 \ldots \ldots \ldots$ & $1500 \pm 200$ & $2.172 \pm 0.002$ & 2.176 & $-400 \pm 300$ & 2.175 & $-300 \pm 300$ \\
\hline
\end{tabular}

Notes. - (2) The full width at half-maximum intensity of the spatially resolved Ly $\alpha$ emission lines shown in Fig. 2 (in $\mathrm{km} \mathrm{s}^{-1}$ ). (3) The mean redshift for the Ly $\alpha$ emission in the nebula (measured from the spectra shown in Fig. 2). (4) The redshift of the narrow "core" of the nuclear Ly $\alpha$ profile (see Fig. 1). The quoted redshifts were calculated from the flux-weighted mean wavelength in a window $\approx 3000 \mathrm{~km} \mathrm{~s}^{-1}$ wide, centered on the peak of the nuclear Ly $\alpha$ profile. These redshifts are uncertain by \pm 0.002 . We do not quote a value for Q $0445+097$ because the nuclear Ly $\alpha$ profile is decimated by very strong absorption (see Fig. 1). (5) The velocity offset between the spatially resolved and narrow nuclear Ly $\alpha$ lines (in $\left.\mathrm{km} \mathrm{s}^{-1}\right)$. Here $\Delta v_{\mathrm{Ly} \alpha} \equiv c\left(z_{\mathrm{Ly} \alpha, \text { neb }}-z_{\mathrm{Ly} \alpha \text {, nuc }}\right) /\left(1+z_{\mathrm{Ly} \alpha \text {, neb }}\right)$. (6) The redshift of the broad nuclear C IV $\lambda 1549$ emission line (uncertain by \pm 0.002$)$. (7) The velocity offset between the spatially resolved Ly $\alpha$ lines and the broad nuclear C IV emission (in $\left.\mathrm{km} \mathrm{s}^{-1}\right)$. Here, $\Delta v_{\mathrm{CIV}} \equiv c\left(z_{\mathrm{Ly} \alpha, \mathrm{neb}}-z_{\mathrm{CIV}, \mathrm{nuc}}\right) /\left(1+z_{\mathrm{Ly} \alpha, \mathrm{neb}}\right)$. 
off-nuclear $L y \alpha$ line. No significant velocity offset is observed for Q0445+097 and Q1318+113. The origin of these offsets may be related to the systematic blueshifts of $C$ IV $\lambda 1549$ with respect to low-ionization BLR lines in high- $z$ quasar spectra (Corbin 1989; Espey et al. 1989).

Three of the QSRs in our sample have nuclear Ly $\alpha$ emission lines with central "self-reversals" (narrow absorption features with $z_{\text {abs }} \approx z_{\text {em }}$ ): Q0445+097, Q0805+046, and Q0941+261. The latter two Ly $\alpha$ absorption lines are rather weak and could be Ly $\alpha$ forest lines. However, it is interesting that in each of these three OSRs, the velocity of the Ly $\alpha$ absorption line agrees with the velocity of the spatially extended Ly $\alpha$ emission line to within about $300 \mathrm{~km} \mathrm{~s}^{-1}$. It is quite possible that the absorbing clouds are closely related to the spatially extended emissionline clouds.

\subsection{He II and Other Emission Lines}

We have not definitely detected any spatially extended emission lines except for $L y \alpha$ in any of the QSR nebulae. We do have likely (formally $\approx 4-5 \sigma$ ) detections of the He II $\lambda 1640$ line in the nebula of Q0445+097 (at $\approx 10 \%$ of $L y \alpha$ ) and the C IV 21549 line in the nebula of Q $0805+046$ (at $\approx 7 \%$ of $L y \alpha$ ): see Fig. 2. Within the spectral region we observed, the strongest lines expected under a wide variety of conditions are $\mathrm{N} \mathrm{v}$ $\lambda 1240$, Si IV $\lambda 1400, \mathrm{C}$ IV $\lambda 1549, \mathrm{He}$ II $\lambda 1640$, and C III] $\lambda 1909$ (though our spectra do not include C III] in Q0805+046 and $\mathrm{Q} 0941+262$ ). Apart from the probable detections noted above, the upper limits for the fluxes of these lines relative to $\mathrm{Ly} \alpha$ range from less than 0.05 for the Q0805+046 and Q1318+113 nebulae to less than 0.25 for the Q0941 + 261 nebula. These are all $3 \sigma$ limits for lines with widths the same as those of the spatially extended $L y \alpha$ lines. These ratios are summarized in Table 3.

Foltz et al. (1988) have observed that the He II $\lambda 1640$ emission line in the nuclei of some high-z QSRs is considerably narrower than the other emission lines. They therefore suggested that this $\mathrm{He}$ II line emission might originate in a region of less-disturbed "extranuclear" gas (possibly the interstellar medium [ISM] of the host galaxy of the QSR). We have confirmed the Foltz et al. empirical result with a significantly larger sample: for 46 high- $z$ QSRs in BTT, the He II lines are on-average only about half as wide as the $\mathrm{C}$ IV $\lambda 1549$ lines (average FWHM of $\approx 3000 \mathrm{~km} \mathrm{~s}^{-1}$ and $\approx 6000 \mathrm{~km} \mathrm{~s}^{-1}$, respectively). These $\mathrm{He}$ II widths are therefore intermediate between the widths of the strong BLR lines and the typical emission-line widths in the QSR nebulae and/or the central regions of high-redshift radio galaxies $\left(\approx 1500 \pm 500 \mathrm{~km} \mathrm{~s}^{-1}\right.$ FWHM; see above and P. McCarthy, 1991, private communication). This suggests that a significant fraction ( $\approx 50 \%$ ?) of the nuclear He II emission in high-z QSRs may arise in the central part of the $L y \alpha$ nebula.

To test this idea, we have compared the equivalent width of the nuclear He II emission line (as measured by BTT) to the equivalent width of the spatially extended Ly $\alpha$ emission (using the luminosities of the Ly $\alpha$ nebulae and QSR UV continuum luminosities measured by HLVM). As can be seen in Figure 4, the two parameters are correlated (at the $97 \pm 2 \%$ confidence level, using the BHK or Cox Hazard tests for data sets containing upper limits; see Isobe, Feigelson, \& Nelson 1986). The

TABLE 3

Line Ratios in the Nebulae and Related Objects

\begin{tabular}{|c|c|c|c|c|c|c|}
\hline $\begin{array}{l}\text { Object } \\
\text { (1) }\end{array}$ & $\begin{array}{l}\text { Ly } \alpha \\
\text { (2) }\end{array}$ & $\begin{array}{l}\mathrm{N} v \\
(3)\end{array}$ & $\underset{\text { (4) }}{\mathrm{Si} I V+\mathrm{O} I V]}$ & $\begin{array}{c}\mathrm{C} \text { IV } \\
(5)\end{array}$ & $\begin{array}{l}\mathrm{He} \text { II } \\
(6)\end{array}$ & $\begin{array}{c}\mathrm{C} \text { III] } \\
(7)\end{array}$ \\
\hline $\mathrm{Q} 0445+097$ & $\equiv 100$ & $<7$ & $<7$ & $<7$ & 10: & $<7$ \\
\hline $\mathrm{Q} 0758+120$ & 100 & $<15$ & $<15$ & $<15$ & $<15$ & $<15$ \\
\hline $\mathrm{Q} 0805+046 \ldots \ldots \ldots \ldots \ldots$ & 100 & $<5$ & $<5$ & 7: & $<5$ & $\ldots$ \\
\hline $\mathrm{Q} 0941+261 \ldots \ldots \ldots \ldots \ldots$ & 100 & $<25$ & $<25$ & $<25$ & & $\ldots$ \\
\hline $\mathrm{Q} 1318+113$ & 100 & $<5$ & $<5$ & $<5$ & $<5$ & 5 \\
\hline Radio galaxies ............. & 100 & 5 & 5 & 12 & 10 & 6 \\
\hline Quasar nucleus ............. & 100 & 26 & 10 & 46 & 5 & 21 \\
\hline Seyfert $2 \ldots \ldots \ldots \ldots \ldots \ldots$ & 100 & 10 & 9 & 21 & 18 & 16 \\
\hline Power law $\ldots \ldots \ldots \ldots \ldots$ & 100 & $<1$ & $<1$ & 3 & 4 & 4 \\
\hline SNRs $\ldots \ldots \ldots \ldots \ldots \ldots$ & 100 & $<1$ & 1 & 2 & 1 & 4 \\
\hline Shock ............. & 100 & $\ll 1$ & 2 & 8 & 1 & 7 \\
\hline Ostar $\ldots \ldots \ldots \ldots \ldots \ldots \ldots$ & 100 & $<1$ & $<1$ & $<1$ & $\leq 2$ & $\leq 6$ \\
\hline Narrow $\% \ldots \ldots \ldots \ldots$ & $25 \%$ & $5 \%$ & $12 \%$ & $6 \%$ & $\equiv 50 \%$ & $7 \%$ \\
\hline
\end{tabular}

Notes.-11) The "radio galaxies" entry is based on a composite spectrum of high-z radio galaxies (objects with redshifts and radio powers similar to our QSRs) constructed by P. McCarthy (1991, private communication). The "Quasar Nucleus" entry is based on a composite broad-line region spectrum for high-z quasars constructed by Boyle 1990. The "Seyfert 2 " entry is the "mean spectrum" adopted by Ferland \& Osterbrock 1986, augmented (when necessary) by the spectrum of NGC 1068 published by Neugebauer et al. 1980. The "Power Law" entry is a model of low-density gas with solar metallicity photoionized by a "power-law" AGN continuum and is taken from Ferland \& Osterbrock 1986. The "SNR" entry comes from three SNRs observed by Benvenuti, Dopita, \& D'Odorico 1980. We have estimated Ly $\alpha$ for these SNRs from the flux of the two photon continuum assuming the low-density limit and $T \approx 10^{4} \mathrm{~K}$. The "Shock" entry is for a $100 \mathrm{~km} \mathrm{~s}^{-1}$ radiative shock from the models of Shull \& McKee 1979. The "O star" entry is from the photoionization models of Stasinska 1990 for $\approx$ solar metallicity and $T_{*}=40,000-45,000 \mathrm{~K}$. The last row ("Narrow \%") is the predicted fraction of the nuclear emission-line flux in a typical QSR that arises in the centralmost part of the nebula (rather than in the BLR proper). This was estimated using the following assumptions: $50 \%$ of the nuclear $\mathrm{He}$ II emission arises in the central nebula, the central nebula has an emission-line spectrum like that of the average high- $z$ radio galaxy (as given in row 6 of this table), and the resulting spectrum (BLR plus central nebula) matches the spectrum of the nucleus of a typical high-z quasar (as given in row 7 of this table). See text for more discussion. (3)-(7) The flux (relative to Ly $\alpha \equiv 100$ ) of the $N v \lambda 1240, S i$ IV + O IV] $\lambda 1400$, C IV $\lambda 1549$, He II $\lambda 1640$, and C III] $\lambda 1909$ lines. 


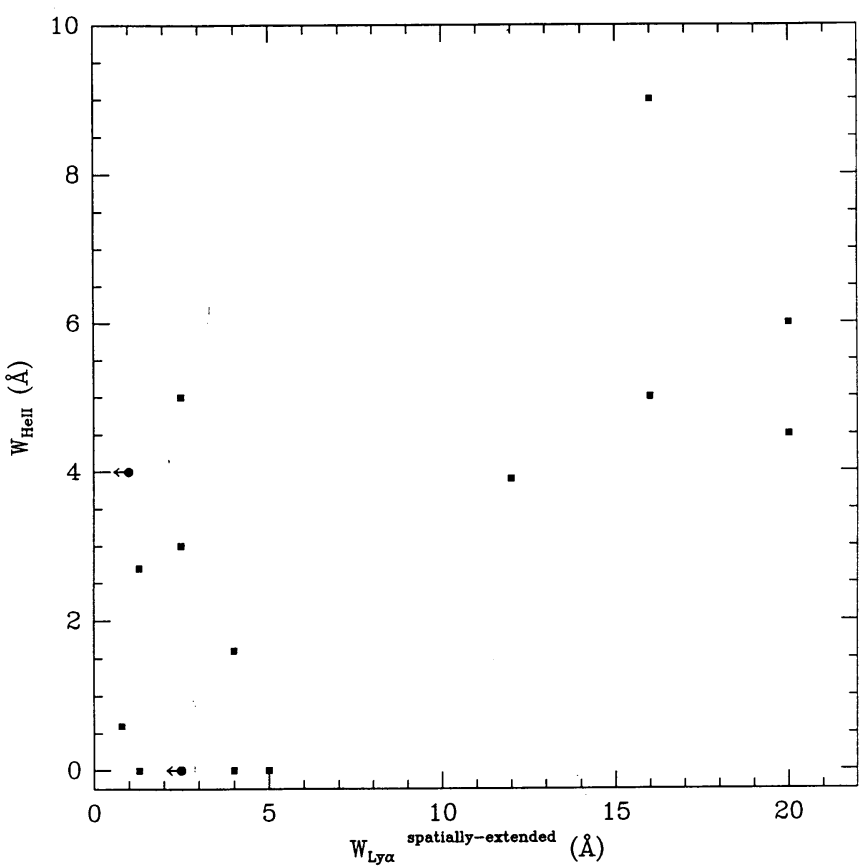

Fig. 4.-The rest-frame equivalent width of the $\mathrm{He}$ II $\lambda 1640$ nuclear emission line (from BTT) is plotted against the rest-frame equivalent width of the spatially extended Ly $\alpha$ emission (see HLVM) for all QSRs common to BTT and HLVM. The latter parameter is defined as the quotient of the spatially resolved Ly $\alpha$ luminosity and the total QSR continuum power per unit wavelength at $\approx 1200 \AA$ in the QSR frame. The correlation is significant at the $\approx 97 \%$ level.

correlation in Figure 4 implies that the nuclear He II $\lambda 1640$ flux is typically $\approx 30 \%$ of the spatially extended Ly $\alpha$ flux. This is consistent with our expectations, since the spatially extended Ly $\alpha$ flux is typically $10 \%$ of the nuclear Ly $\alpha$ flux in high-z QSRs (HLVM), while the $\mathrm{He} \mathrm{II} / \mathrm{Ly} \alpha$ ratio is typically $5 \%$ in QSR nuclei (see Boyle 1990; BTT).

Thus, we find suggestive evidence supporting the conjecture of Foltz et al. (1988) that the relatively narrow nuclear He II $\lambda 1640$ lines in high- $z$ QSRs are associated with the presence of strong, spatially extended emission-line nebulosity. Selecting by the presence of strong, narrow He II $\lambda 1640$ lines should be a good way to find more high-z quasars with prominent Ly $\alpha$ nebulae.

\section{DISCUSSION}

\subsection{Physical Properties and Cloud Confinement}

In HLVM we derived expressions for the densities, masses, and filling factors of the Ly $\alpha$-emitting material in the QSR nebulae under the assumption that the nebulae consisted of clouds photoionized by the QSR continuum. These expressions were all written in terms of the ionization parameter $U \equiv Q_{\text {ion }} /\left(4 \pi r^{2} n c\right)$ for a cloud with number density $n$ located a distance $r$ away from an source of luminosity $Q_{\text {ion }}$ photons s ${ }^{-1}$. If the likely C IV $\lambda 1549$ detection in the Q0805 + 046 nebula is genuine, then standard AGN photoionization models (Stasinska 1984; Ferland \& Netzer 1983) require $\log U \geq-2.3$ to produce C IV emission that is stronger than C III] $\lambda 1909$. The general arguments given in Krolik, McKee, \& Tarter (1981) limit $\log U \leq-1$ for clouds photoionized by an AGN (to avoid runaway heating to the Compton temperature). For $\log U \approx-2$ (consistent with these limits for Q0805+046 and equal to the value providing the best match to the radio galaxy spectrum given in Table 3), the expressions in HLVM imply that the parameters appropriate to the Ly $\alpha$-emitting material ("clouds") in the Q0805+046 nebula are densities in the clouds $n_{e} \approx 100 r_{23}^{-2} \mathrm{~cm}^{-3}$ (where $r$ is in units of $10^{23} \mathrm{~cm} \approx 30$ kpc), pressures $P \approx 3 \times 10^{-10} r_{23}^{-2}$ dyne $\mathrm{cm}^{-2}$, total Ly $\alpha-$ emitting cloud mass $M_{c} \approx 10^{9} M_{\odot}$, volume filling factor of the clouds $\approx 10^{-6}$, thickness of the ionized layer of each cloud $1_{\mathrm{H} \text { II }} \approx 10^{19} r_{23}^{2} \mathrm{~cm}$.

Following arguments given in Crawford \& Fabian (1989), HLVM argued that the Ly $\alpha$-emitting clouds are likely to be confined by some intercloud medium (ICM), in order to avoid excessively large cloud-creation rates that would be needed to balance cloud photoevaporation. An ICM could confine the clouds by static thermal pressure or by dynamic (ram) pressure. The cloud velocities we observe are $\sigma_{3 D} \approx\left(3 \sigma_{\text {los }}\right)^{1 / 2} \approx$ $10^{3} \mathrm{~km} \mathrm{~s}^{-1}$, where $\sigma_{\text {los }}$ and $\sigma_{3 \mathrm{D}}$ are the line-of-sight and threedimensional velocity dispersions implied by typical Ly $\alpha$ FWHMs of $1000-1500 \mathrm{~km} \mathrm{~s}^{-1}$. These velocities correspond to a Mach number $M \approx 2.2 T_{7}^{-1 / 2}$ where $T_{7}$ is the ICM temperature in units of $10^{7} \mathrm{~K}$. Since the virial temperature for the ICM in a galaxy potential is $\leq 10^{7}$, the cloud motions through such an ICM will likely be supersonic, and ram pressure will be important (unless the clouds and the ICM are comoving). Assuming the cloud temperatures are set by photoionization $\left(T \approx 10^{4} \mathrm{~K}\right)$, ram pressure confinement of the clouds in Q0805 + 046 requires an ICM ion number density $n_{\mathrm{ICM}} \approx$ $0.02 r_{23}^{-2} \mathrm{~cm}^{-3}$. The total implied ICM mass out to a radius $r_{23}$ is $M_{\mathrm{ICM}}\left(r<r_{23}\right) \approx 2 \times 10^{11} r_{23} M_{\odot}$. If the clouds and the ICM are comoving, the ICM mass implied by static thermal confinement of the clouds is about $\approx 10^{12} r_{23} T_{7}^{-1} M_{\odot}$.

\subsection{Kinematics and Dynamics of the Nebulae}

The kinematics of the Ly $\alpha$ nebulae are clearly extraordinary compared to normal galaxies, but the physical origin of the kinematics is unclear. Because $\operatorname{Ly} \alpha$ photons are so susceptible to radiative transfer effects, the assumption that the Ly $\alpha$ profiles can be used reliably to trace the motions of the ionized gas in these nebulae may be incorrect (e.g., Urbaniak \& Wolfe 1981; Neufeld 1990; Neufeld \& McKee 1988; and see $\S 4.7$ below). If we nevertheless adopt this assumption, two general possibilities are that the gas motions have a gravitational origin or that they arise through energetic processes associated with the QSR (e.g., acceleration by radiation pressure, by a thermal wind, or by the outflowing radio plasma).

Considering the first possibility, we note (as a point of reference) that the typical line-of-sight velocity dispersion of a giant elliptical galaxy is $\sigma_{\text {los }} \approx 300 \mathrm{~km} \mathrm{~s}^{-1}$, corresponding to a characteristic line width (FWHM) of $\approx 700 \mathrm{~km} \mathrm{~s}^{-1}$. In contrast, the Ly $\alpha$ line widths in the QSR nebulae are significantly greater than this. The dynamics of the $\operatorname{Ly} \alpha$ nebulae are then unlikely to be simple orbital motion in the potential well of a galaxy. The maximum plausible gravitationally induced velocities will arise when gas falls freely into a galaxy from large radii. Such a situation might occur during the process of galaxy formation (e.g., Fall \& Rees 1985; Chevalier \& Lufkin 1990). Taking a potential of the form $\Phi(r)=v_{\text {circ }}^{2} \ln \left(r / r_{\max }\right)$ e.g., an isothermal potential with an outer cutoff at $r_{\max }$ and a depth corresponding to a circular orbital velocity $v_{\text {circ }}(r)$-yields the relation $\sigma_{\text {los }}^{2}=\frac{1}{3} v_{\text {circ }}^{2}$ for a galaxy with a Hubble profile. This then implies an infall velocity $v_{\text {in }}=\left(\begin{array}{ll}6 & \sigma_{\text {los }}\end{array}\right)^{1 / 2}\left[\ln \left(r_{\max } / r\right)\right]^{1 / 2}$. For $\sigma_{\text {los }} \approx 300 \mathrm{~km} \mathrm{~s}^{-1}$, this implies $v_{\text {in }} \approx 600 \mathrm{~km} \mathrm{~s}^{-1}$ for $r=$ $r_{\text {max }} / 2$ and $v_{\text {in }} \approx 1100 \mathrm{~km} \mathrm{~s}^{-1}$ for $r=r_{\text {max }} / 10$. Allowing for the 
front and back sides of the infall (redshifted and blueshifted gas, respectively), line widths comparable to those seen in the QSR nebulae are plausible.

An outflow driven by the QSR or its radio source is also plausible, especially since some radio and Seyfert galaxies show fairly clear evidence for the acceleration of emission-line clouds by the radio plasma (e.g., van Breugel et al. 1985, 1986; Whittle et al. 1988). Indeed, the characteristic dynamical time scale for the QSR nebulae $\left(\approx 10^{8} \mathrm{yr}=\right.$ diameter divided by internal velocity dispersion) is similar to the ages inferred indirectly for the radio sources associated with high- $z$ radio galaxies (e.g., Chambers et al. 1990). The line widths of $\approx 1000$ $1500 \mathrm{~km} \mathrm{~s}^{-1}$ that we observe over scales of tens of kiloparsecs in the QSR nebulae are reasonable for such a process (as we now demonstrate).

As an rough approximation, we assume that the radio plasma inflates a spherical bubble in the ISM of the QSR (see Begelman \& Cioffi 1989 and Chambers 1989 for more detailed nonspherical calculations). Castor, McCray, \& Weaver's (1975) classic treatment of an energy-conserving bubble being inflated by a source of mechanical energy $L$ into a uniform medium with proton number density $n$ yields the following relationship between the bubble's radius and expansion velocity: $v \approx$ $510 L_{46}^{1 / 3} r_{23}^{-2 / 3} n^{-1 / 3} \mathrm{~km} \mathrm{~s}^{-1}$, where $L$ is in $10^{46} \mathrm{ergs} \mathrm{s}^{-1}, r$ is in $10^{23} \mathrm{~cm}(\approx 30 \mathrm{kpc})$, and $n$ is in $\mathrm{cm}^{-3}$. Eliminating $n$ in favor of the total interstellar mass interior to $r$, we obtain $v \approx$ $770 L_{46}^{1 / 3} M_{12}^{-1 / 3} r_{23}^{1 / 3} \mathrm{~km} \mathrm{~s}^{-1}$, where $M$ is in $10^{12} M_{\odot}$. Thus, for the maximum plausible ISM mass for a giant protogalaxy and for a jet mechanical luminosity large enough to power the observed radio synchrotron luminosities (all within a factor of 2 of $5 \times 10^{45}$ ergs s $^{-1}$ for these QSRs; see HLVM), we obtain typical expansion velocities that are compatible with the observed linewidths.

As one test of the idea that the gasdynamics are driven by the radio source, we have searched for a relationship between the proximity of radio plasma and the presence of strong velocity gradients and/or broad Ly $\alpha$ emission lines. Our spectrograph slit was nearly along the radio axis for Q0941+262 and $\mathrm{Q} 1318+113$, was $\approx 30^{\circ}$ offset for $\mathrm{Q} 0445+097$ and $\mathrm{Q} 0805+046$, and was $\approx 50^{\circ}$ offset for $\mathrm{Q} 0758+120$. We see no trend in the Ly $\alpha$ line widths along this sequence. Moreover, the gas around Q1318 + 113 appears to be somewhat more kinematically disturbed on the side of the QSR where the radio emission is considerably weaker (the Ly $\alpha$ line width is $1200 \pm 200 \mathrm{~km} \mathrm{~s}^{-1}$ on the side of the strong radio emission and $1900 \pm 400 \mathrm{~km} \mathrm{~s}^{-1}$ on the other side). Unfortunately, this is the only QSR in the present sample for which we can make this test, as it is the only one with a very asymmetric radio source and readily detectable Ly $\alpha$ emission on both sides of the QSR). We conclude that there is no obvious kinematic evidence for acceleration by the radio source in these QSRs. A larger sample and more complete mapping of the kinematics in these nebulae are both needed to test this idea further.

For the cloud mass estimated in $\S 4.1$ for Q0805+046, the total kinetic energy in the clouds is $\approx 10^{58}$ ergs. We have argued in HLVM (and see $\S 4.1$ and 4.7 below) that the total interstellar mass associated with the high- $z$ QSR nebulae is likely to be $\approx 10^{11}-10^{12} M_{\odot}$ (for an ICM sufficient to confine or replenish the clouds over the radio source lifetime). If the high velocities we observe in $L y \alpha$ pertain to the rest of the interstellar matter, the total kinetic energy would be $\approx 10^{60}$ $10^{61}$ ergs. This is roughly equal to both the energy content of the radio plasma (assuming standard "minimum energy" con- ditions; e.g., Miley 1980) and the $P \Delta V$ work implied by the inferred radial pressure profiles in the Ly $\alpha$ nebulae (see HLVM and $\S 4.1$ above). These energies exceed the total gravitational binding energy of a typical galaxy by about an order of magnitude.

\subsection{The Nuclear He II Emission}

If much of the He II emission seen toward the nuclei of high- $z$ QSRs does in fact arise in the inner (arcsec-scale) part of the Ly $\alpha$ nebula (as we have suggested in $\S 3.3$ above), a necessary consequence would be that much of the centralmost few thousand $\mathrm{km} \mathrm{s}^{-1}$ of the nuclear Ly $\alpha$ emission-line profile is directly associated with this "narrow" He II emission. We suggested this in $\S 3.2$ above on the basis of the kinematic continuity between the core of the nuclear Ly $\alpha$ profile and the Ly $\alpha$ nebular emission. While a "narrow" component should also be present in the nuclear profiles of the UV metal lines (e.g., C IV $\lambda 1549$ and $C$ III] $\lambda 1909$ ), it may be undetectably weak relative to the BLR emission. Clearly, if our hypothesis about He II is correct, the narrowness of $\mathrm{He}$ II relative to other strong lines in the nuclei of QSRs can be understood only if the nebula makes a significantly greater relative contribution to the nuclear $\mathrm{He}$ II line than to other strong nuclear lines (e.g., C IV 21549). We now show that this is likely to be the case.

In high-redshift radio galaxy nebulae, the C III] $\lambda 1909$ and $\mathrm{C}$ Iv $\lambda 1549$ lines are about as strong as $\mathrm{He}$ II $\lambda 1640$ (P. McCarthy, private communication; see Table 3 ). In contrast, the $\mathrm{C}$ III] and $\mathrm{C}$ IV lines are about 5-10 times stronger than $\mathrm{He}$ II in the BLR of quasars (see Boyle 1990 and Table 3). Thus, if the QSR nebulae have emission-line spectra similar to the radio galaxy nebulae, it would be very difficult to detect any $\mathrm{C}$ III] or C IV analog to the "narrow" He II lines in the QSR nuclei (the nuclear C III] and C IV emission lines would be dominated by the BLR). We have quantified these arguments in Table 3 , in which it can be seen that if - for example- $50 \%$ of the nuclear He II emission arises in the inner nebula (and 50\% in the BLR proper), and if the inner nebula is assumed to have the same line ratios as the high- $z$ radio galaxy nebulae, then the predicted fraction of the nuclear emission-line fluxes arising in the inner nebula ranges from only about $6 \%$ for $\mathrm{N} v \lambda 1240, \mathrm{C}$ IV $\lambda 1549$, and C III] $\lambda 1909$ to $25 \%$ for Ly $\alpha$. Thus (empirically) the He II $\lambda 1640$ line would seem to be the UV line most sensitive to the presence of a nebula.

Standard AGN photoionization models predict that the He II $\lambda 1640$ line will have a rest-frame equivalent width $(\mathrm{REW}) \approx 40 \pm 15(\Omega / 4 \pi) \AA$ (where $\Omega$ is the solid angle in steradians subtended by the material as seen from the nucleus). Since the average REW of the He II $\lambda 1640$ emission line is $\approx 5$ $\AA$ in the large sample of QSRs studied by BTT, the implied covering factor of the $\mathrm{He}$ II emitting material is $\approx 10 \%-20 \%$. The nuclear $\mathrm{He}$ II line has an average width that is intermediate between that of strong lines in the BLR and the spatially extended $\mathrm{Ly} \alpha$ emission lines ( $\langle\mathrm{FWHM}\rangle$ of $6000 \mathrm{~km} \mathrm{~s}^{-1} \mathrm{vs}$. $3000 \mathrm{~km} \mathrm{~s}^{-1}$ vs. $1250 \mathrm{~km} \mathrm{~s}^{-1}$ ). This suggests that only a portion $(\approx$ half?) of the nuclear He II line is associated with the inner part of the Ly $\alpha$ nebula. The typical covering factor of this material would then be $5 \%-10 \%$.

\subsection{The $z_{\text {abs }} \approx z_{\text {em }}$ Systems}

One of the goals of the work discussed in HLVM and the present paper was to search for and investigate an emissionline counterpart to the $z_{\mathrm{abs}} \approx z_{\mathrm{em}}$ systems seen in some high-z QSRs (e.g., BTT; Foltz et al. 1988; Anderson et al. 1987). We 
believe that there are three lines of evidence that the Ly $\alpha$ nebulae (and the nuclear He II $\lambda 1640$ emission lines) are related to the $z_{\mathrm{abs}} \approx z_{\mathrm{em}}$ systems. First, in their classic study of the $z_{\text {abs }} \approx z_{\text {em }}$ system in 3C 191, Williams et al. (1975) calculated that the absorbing material arose in gas with a density of $\approx 10^{3}$ $\mathrm{cm}^{-3}$ located $\approx 10 \mathrm{kpc}$ from the QSR nucleus. This is very similar to the properties we measured or deduced for the Ly $\alpha$ nebulae (see HLVM and $\S 4.1$ above).

Second, the covering factor of the emission-line gas in the core of a typical Ly $\alpha$ nebula (estimated above as $5 \%-10 \%$ from the "narrow" nuclear He II $\lambda 1640$ emission line) is similar to the incidence rate of the $z_{\mathrm{abs}} \approx z_{\mathrm{em}}$ systems in high- $z$ QSRs. BTT have cataloged and identified absorption lines seen in the spectra of 67 high- $z$ QSRs. Of the $68 \mathrm{C}$ IV doublets they identify, 14 have REW $>2 \AA$ and nearly all of these (11 of 14) have velocities within about $2000 \mathrm{~km} \mathrm{~s}^{-1}$ of the QSR C IV emission line (compared to only 10 of the $54 \mathrm{C}$ IV doublets with REW $\leq 2 \AA$ ). There seems therefore little doubt that the 11 systems with C IV REW $>2 \AA$ and $|\Delta v| \leq 2000 \mathrm{~km} \mathrm{~s}^{-1}$ are physically associated with the QSRs. Their incidence rate is therefore $11 / 68 \approx 16 \pm 5 \%$. We note parenthetically that the implied covering factors of the "narrow" He II $\lambda 1640$ emitters and $z_{\text {abs }} \approx z_{\text {em }}$ systems are similar to the fraction of high- $z$ QSRs with strongly distorted radio sources, consistent with the idea that the distortion is caused by a collision between the radio jet and gas clouds in the QSR halo (cf. Miley 1987).

Third, the typical velocity differences between the absorbers and the QSR emission lines are consistent with the line widths observed in the Ly $\alpha$ nebulae. For the $11 z_{\text {abs }} \approx z_{\mathrm{em}}$ systems in BTT discussed above, the median velocity difference between the absorption lines and the QSR emission-line redshift is $\approx 500 \mathrm{~km} \mathrm{~s}^{-1}$ (compared to the average FWHM of $1250 \mathrm{~km}$ $\mathrm{s}^{-1}$ in the Ly $\alpha$ nebulae). Note that we have used the $\mathrm{C}$ III] $\lambda 1909$ and He II $\lambda 1640$ lines tabulated in BTT to define the QSR emission-line redshift (the nuclear Ly $\alpha$ profile is affected by Ly $\alpha$ forest absorption of its blue side, and the C IV $\lambda 1549$ line is often strongly blueshifted with respect to the lower ionization lines: cf. Corbin 1989; Espey et al. 1989; our Table 2 and the discussion in $\S 3.2$ ).

We suggest that the cleanest way to determine the interrelationship between the spatially extended Ly $\alpha$ nebulae, the "narrow" nuclear He II $\lambda 1640$ emission, and the $z_{\mathrm{abs}} \approx z_{\mathrm{em}}$ systems would be to obtain long-slit near-IR spectra of forbidden-line emission from high-z QSRs: if much of the "narrow" He II emission really arises in relatively low-density gas with a covering factor large enough to explain the incidence rate of the $z_{\mathrm{abs}} \approx z_{\mathrm{em}}$ systems, we should see strong (one to several times as strong as the He II $\lambda 1640$ line) [O II] $\lambda 3727$ and [O III] $\lambda 5007$ emission lines with widths of greater than $10^{3} \mathrm{~km} \mathrm{~s}^{-1}$. This is consistent with data on radio galaxies at $z>1$, which have [O II] $\lambda 3727$ lines with FWHM $\approx 1400 \mathrm{~km}$ $\mathrm{s}^{-1}$ and a flux ratio [O $\left.\mathrm{II}\right] \lambda 3727 / \mathrm{He}$ II $\lambda 1640 \approx 1$ (P. McCarthy, 1991, private communication).

The associated C IV absorbers listed in BTT are equally likely to exhibit both red and blue shifts with respect to the nuclear C III] $\lambda 1909$ and He II $\lambda 1640$ lines. This might suggest that the associated absorbers are clouds in a highly turbulent velocity field (or perhaps in the halos of galaxies in a cluster associated with the QSR; see Weymann et al. 1979). However, the broad nuclear emission-line profiles may systematically misrepresent the true QSR systemic velocity (e.g., Corbin 1989; Espey et al. 1989). It would therefore be interesting to compare the redshifts of the absorbers to the mean redshift of the spa- tially extended gas in the Ly $\alpha$ nebulae. The only QSR for which this can be done so far is Q0445+ 097. The C IV $\lambda 1549$ absorption in this case is blueshifted by $\approx 350 \pm 150 \mathrm{~km} \mathrm{~s}^{-1}$ relative to the spatially extended $\operatorname{Ly} \alpha$ emission (weakly favoring outflow in this case). It is clearly important to perform this test on a suitably large sample of QSRs.

\subsection{The Ionization of the $\operatorname{Ly} \alpha$ Nebulae}

In HLVM we argued that the Ly $\alpha$ nebulae were most likely photoionized by the high-energy continua of the central QSRs. This argument was based on simple energetics: the luminosity of the observed QSR UV continua is typically about two orders of magnitude greater than the amounts required to power the spatially resolved Ly $\alpha$ emission and typically one order of magnitude (or more) greater than the luminosity of the spatially resolved source of UV continuum emission (possibly due to hot stars). We can now reexamine this issue using the spectra presented in this paper.

In Table 3, we compare the relative strengths of the principal UV emission lines contained within our spectral window for a variety of objects (our QSR nebulae, high-z radio galaxies, the nuclei of typical quasars, type 2 Seyfert nuclei, and supernova remnants) and models (photoionization of gas by a "standard" AGN continuum, heating by shocks, photoionization by typical $O$ stars). Details concerning the data and models may be found in the notes to Table 3 . It is clear that the present data cannot rule out any class of model, though the QSR nebulae are significantly different from the nuclei of AGNs (where the densities are thought to be high enough to suppress collisionally the primary coolants - the optical forbidden linesthereby increasing the temperature and the strength of the collisionally excited UV permitted and semiforbidden lines).

AGN photoionization models predict that $\mathrm{He}$ II $\lambda 1640$ should be at least several percent of $\mathrm{Ly} \alpha$. This arises from simple Zanstra-type photon-counting arguments. Thus, it is a much more robust prediction than the strengths of the collisionally excited metal lines and holds over a broad range in density, ionization parameter, and metal abundance (e.g., Stasinska $1984 ; 1990)$. The likely detection of He II $\lambda 1640$ in the Q0445 + 097 nebula notwithstanding, if future data show that this line is weaker than this, other ionization sources may be favored. If the detection of $\mathrm{He}$ II $\lambda 1640$ can be confirmed, it will rule out photoionization by massive stars, since He II $\lambda 1640$ is predicted to be $\ll 1 \%$ of $\mathrm{Ly} \alpha$, even for stars as hot as $55,000 \mathrm{~K}$ (Stasinska 1990).

\subsection{Metallicity Constraints}

If the likely detection of the spatially extended C IV $\lambda 1549$ emission line in Q0805 + 046 can be confirmed, what inferences could we make about metallicities? Models of gas photoionized by a hard AGN continuum show that the strengths of the C IV $\lambda 1549$ and C III] $\lambda 1909$ lines (relative to $L y \alpha$ ) are relatively insensitive to metallicity over the range from several percent of solar to several times solar (Stasinska 1984; Ferland \& Netzer 1983). This is because these collisionally excited metal lines are important coolants for the gas (i.e., decreasing the metallicity does not significantly affect the strengths of these lines until the metallicity drops so low that collisionally excited $\mathrm{H}$ I emission becomes the dominant coolant). At the extremely low metallicity of $10^{-3}$ solar, the $\mathrm{C} \mathrm{III]/Ly} \alpha$ and $\mathrm{C} \mathrm{IV/Ly} \alpha$ ratios are lowered by a factor of $\approx 10^{2}$ relative to their values for solar metallicity (T. Kallman, private communication). Thus, if the 
C IV line in Q0805+046 is a bona fide detection, this QSR nebula cannot have an extreme Population II metallicity.

\subsection{Scattering Models for the $\mathrm{Ly} \alpha$}

Might the $\operatorname{Ly} \alpha$ nebulae be giant reflection nebulae rather than intrinsic sources of Ly $\alpha$ (cf. Fabian 1989; Scarrott, Rolph, \& Tadhunter 1990)? We can immediately rule out dust scattering or Thompson scattering of the bright QSR nuclear light because the nebular spectra are so different from the nuclear spectra. Insofar as we have not detected (with absolute certainty) spatially extended emission lines other than Ly $\alpha$, we now consider the possibility that the spatially extended Ly $\alpha$ is produced by resonant scattering of the nuclear (BLR) Ly $\alpha$ photons by $\mathrm{H}$ I in the QSR halos.

If the QSR halo contains clouds that are optically thick to the Lyman continuum, then it is clear that Ly $\alpha$ production by photoionization and recombination will dominate over resonant scattering in these clouds: the nuclei of our QSRs emit (typically) about 30 times more Lyman continuum photons than photons with frequencies appropriate for $L y \alpha$ resonant scattering (given the observed velocity dispersions in the nebulae).

The resonant scattering model can then only be relevant if the medium producing the spatially extended Ly $\alpha$ emission is highly optically thin to Lyman continuum radiation from the QSR. Consider an individual cloud of gas in the QSR halo. In the extreme case that it is optically thin to $\operatorname{Ly} \alpha$ (as well as to the Lyman continuum), the ratio of $\mathrm{Ly} \alpha$ photons produced by resonant scattering to those produced by photoionization and recombination is $N_{\mathrm{sc}} / N_{\mathrm{rec}} \approx\left(\sigma_{\mathrm{Ly} \alpha} / \sigma_{0}\right)(L / C)\left(2 v_{\text {therm }} / c\right)(\alpha+3)$, where $\sigma_{\mathrm{Ly} \alpha}$ and $\sigma_{0}$ are the absorption cross sections at the center of $\operatorname{Ly} \alpha$ and at the Lyman edge respectively, $L / C$ is the ratio of the photon densities (photons $\mathrm{Hz}^{-1}$ ) incident on the cloud at Ly $\alpha$ and at the Lyman edge, $v_{\text {therm }}$ is the thermal velocity of $\mathrm{H}_{\mathrm{I}}$ in the cloud, and $\alpha$ is the power-law index of the incident spectrum $\left(P_{v} \approx v^{-\alpha}\right)$. Since $\sigma_{\text {Ly } \alpha} / \sigma_{0} \approx 10^{4}\left(T / 10^{4}\right.$ $\mathrm{K})^{-1 / 2}$ and $v_{\text {therm }} / c \approx 4 \times 10^{-5}\left(T / 10^{4} \mathrm{~K}\right)^{1 / 2}$, then $N_{\text {sc }} / N_{\text {rec }} \approx$ $(L / C)(\alpha+3) \approx 10$ for our typical QSRs.

As a particular example of a case in which scattering might exceed recombination, suppose that the high- $z$ QSRs are immersed in hot atmospheres analogous to those surrounding X-ray-luminous giant elliptical galaxies (e.g., Fabian 1989). The electron column density to the nucleus of M87 is $N_{e} \approx 4$ $\times 10^{21} \mathrm{~cm}^{-2}$ (Stewart et al. 1984). For gas dominated by collisional ionization, the neutral fraction is $n_{0} / n_{e} \approx 10^{-8} T_{7}^{-1.25}$ (where $T_{7}$ is the temperature in $10^{7} \mathrm{~K}$ ). Photoionization equilibrium gives $n_{0} / n_{e} \approx 2 \times 10^{-9} Q_{57}^{-1} r_{23}^{2}\left(n_{e} / 10^{-2}\right) T_{7}^{-0.75}$, where $Q_{57}$ is the Lyman continuum luminosity of the photoionization source (in units of $10^{57} \mathrm{~s}^{-1}$ ) and $r_{23}$ is the distance from this source (in units of $10^{23} \mathrm{~cm}$ ). Taking parameters appropriate to our QSRs $\left(Q_{57} \approx 1 ; r_{23} \approx 1\right)$ and M87's gaseous halo $\left(r_{23}^{2}\left[n_{e} / 10^{-2}\right] \approx 1 ; T_{7} \approx 1\right)$, we estimate an $\mathrm{H}$ I column density of $N_{0} \approx 10^{13} \mathrm{~cm}^{-2}$. This corresponds to an optical depth in Ly $\alpha$ of $\tau_{\mathrm{Ly} \alpha} \approx 0.02 T_{7}^{-1 / 2}$. Such a halo could then produce resonantly scattered Ly $\alpha$ emission with a luminosity of about $2 \%$ of the luminosity of the centralmost $\approx 10^{3} \mathrm{~km} \mathrm{~s}^{-1}$ of the nuclear Ly $\alpha$ line. This is about an order of magnitude smaller than the observed luminosities of the Ly $\alpha$ nebulae (see HLVM), but increasing the value for $r_{23}^{2}\left[n_{e} / 10^{-2}\right]$ to $\approx 10$ (as suggested by Fabian for the halos of high-z QSRs) would increase $\tau_{\text {Ly } \alpha}$ to $\approx 0.1$ (consistent with the observed luminosities of the nebulae). The implied mass of hot gas out to a radius of $50 \mathrm{kpc}$ from the QSR would be of order $10^{12} M_{\odot}$ in this case.

\subsection{Comparison to High-z Radio Galaxies}

One of the major quests of present AGN research is to understand the relationship among the multitude of AGN classes. There are several "unification schemes" for AGNs that maintain that QSRs and radio galaxies are related through either viewing angle effects or evolution (e.g., Barthel 1989; Norman \& Miley 1984). Observations of the properties of the spatially resolved material in the two types of objects make it possible to test these hypotheses. As we discussed in HLVM, the basic global properties of the QSR Ly $\alpha$ nebulae (e.g., luminosity and size) are quite similar to those of the Ly $\alpha$ emission associated with radio galaxies having similar redshifts, radio powers, and radio spectra to the QSRs; (cf. McCarthy 1988, Chambers 1989, and references therein). Our new spectroscopic data allow us to compare the kinematics and physical state of the high- $z$ QSR and high- $z$ radio galaxy nebulae.

To first order, the kinematics of the two types of Ly $\alpha$ nebulae are similar: the gas is very kinematically active with characteristic internal velocities of order $10^{3} \mathrm{~km} \mathrm{~s}^{-1}$. More detailed comparisons are not yet possible because there are so few members of either class with well-studied kinematics. There is, however, a hint that the two types of nebulae may be kinematically different. While none of the QSR nebulae show any velocity shears in excess of $500 \mathrm{~km} \mathrm{~s}^{-1}$, some radio galaxy nebulae show well-organized large-scale velocity shears with much larger amplitudes. Specifically, the nebulae associated with the high- $z$ radio galaxies 3C 294 (McCarthy et al. 1990), 3C 324 (Spinrad \& Djorgovski 1984), and 3C 326.1 (McCarthy et al. 1987) exhibit organized shears with amplitudes of $\approx 1000-1500 \mathrm{~km} \mathrm{~s}^{-1}$ over distances of $\approx 50-100 \mathrm{kpc}$. The nebula associated with the radio galaxy $4 \mathrm{C} 41.17$ at $z=3.8$ also exhibits large velocity shears, though the kinematics appear more complex than those of the three above galaxies (Chambers et al. 1990). While the nebula in the high- $z$ radio galaxy 3C 368 (Djorgovski et al. 1987a) has a velocity field with a relatively small amplitude $\left(\approx 500 \mathrm{~km} \mathrm{~s}^{-1}\right)$, the emission lines are also very narrow $\left(F W H M \approx 200-500 \mathrm{~km} \mathrm{~s}^{-1}\right.$ ). To our knowledge, there is no high- $z$ radio galaxy nebula which simultaneously shows the broad lines $\left(\approx 1000-1500 \mathrm{~km} \mathrm{~s}^{-1}\right.$ FWHM) and low-amplitude velocity shears $\left(<500 \mathrm{~km} \mathrm{~s}^{-1}\right)$ seen in the five QSR nebulae.

If this kinematic difference is confirmed by studies of larger samples, it would have interesting implications for "viewing angle type" unification schemes (e.g., Barthel 1989). If these gradients are interpreted as arising in bipolar outflows driven by, and coaxial with, the radio source (e.g., McCarthy et al. 1990), it is surprising that the radio galaxies (where Barthel's model predicts that the outflow should be predominantly in the plane of the sky) would show larger velocity shears than the QSRs (where the outflow should be predominantly along the line of sight).

We can also compare the strengths of the other emission lines relative to $\mathrm{Ly} \alpha$ in the QSR and radio galaxy nebulae. P. McCarthy (1991, private communication) has studied the spectra of 10 high- $z$ radio galaxies and found that the $\mathrm{N} v$ $\lambda 1240$, Si IV $\lambda 1400, \mathrm{C}$ IV $\lambda 1549, \mathrm{He}$ II $\lambda 1640$, and C III $\lambda 1909$ emission lines are typically $5 \%, 5 \%, 12 \%, 10 \%$, and $6 \%$ as strong as $\mathrm{Ly} \alpha$, respectively (with typical variations of a factor of 2 in these line ratios from object to object). McCarthy et al. $(1987,1990)$ give further details concerning the spatial extent of some of these lines. These line ratios are loosely consistent with the likely detections and upper limits in Table 3 for the QSR nebulae for which we have the highest signal-to-noise data (Q0445 + 097, Q0805+046, and Q1318+113). 


\subsection{Comparison to Cooling Flow Nebulae}

The largest and brightest emission-line nebulae in the present-day universe are the so-called "cooling-flow nebulae" (see Hu, Cowie, \& Wang 1985; Heckman et al. 1989). Fabian and collaborators have argued that the emission-line nebulae associated with QSRs are in fact cooling flows that are illuminated by the QSR ionizing continuum (see Crawford \& Fabian 1989, Forbes et al. 1990, and references therein). It is therefore of interest to compare the properties of our high-z QSR Ly $\alpha$ nebulae to those of well-studied low- $z$ cooling flow nebulae.

The latter have typical $e$-folding length scales of a few kiloparsecs or less and typical estimated or observed Ly $\alpha$ luminosities of $10^{41}$ to few times $10^{42} \mathrm{ergs} \mathrm{s}^{-1}$ ( Hu 1988; Heckman et al. 1989). The high- $z$ QSR nebulae are therefore typically an order of magnitude larger and more than two orders of magnitude more luminous than the low- $z$ cooling flows (not surprising if the former are photoionized by the extremely powerful QSR continuum). The kinematics of the cooling flow and QSR nebulae show a striking qualitative similarity in that both are characterized by a lack of well-organized large-scale shears (Heckman et al. 1989). However, the typical line widths are several times larger in the high- $z$ QSR nebulae (1250 \pm 250 $\mathrm{km} \mathrm{s}^{-1}$ vs. $370 \pm 150 \mathrm{~km} \mathrm{~s}^{-1}$ for the characteristic velocity scales in the two respective classes).

The quantitative inferences about ICM densities, masses, cooling times, and cooling/inflow rates in high-z QSRs drawn by Fabian and colleagues are based on the assumption that the pressure that is estimated from the emission-line clouds is balanced by the static thermal pressure of a hot ICM with a temperature near the virial temperature of a massive galaxy $\left(\approx 10^{7} \mathrm{~K}\right)$. As we have noted in $\S 4.1$ above, the typical cloud motions in the nebulae are $\approx 10^{3} \mathrm{~km} \mathrm{~s}^{-1}$, corresponding to a Mach number of $M \approx 2.2 T_{7}^{-1 / 2}$ for an ICM with a temperature in units of $10^{7} \mathrm{~K}$. If an ICM with $T_{7} \approx$ unity is indeed present, the cloud pressure is more likely set by ram pressure than static thermal pressure. Thus, the quantitative details of the picture developed by Fabian and colleagues may need to be modified for the high-z QSRs we have investigated (even though the basic qualitative picture may be valid).

\section{CONCLUSIONS}

As we described in $\S 1$, the study of the environments of high-redshift radio-loud quasars (QSRs) bears on a number of interesting issues in extragalactic astronomy. In closing, we will summarize the implications of our results for these issues.

Inferences drawn from our data about the processes involved in the formation and early evolution of galaxies are very uncertain because the QSR itself may have already obliterated any signature of these processes. If the detection of the spatially extended C IV $\lambda 1549$ line can be confirmed, it will imply that metal-enriched (nonprimordial) material is already present over galactic-scale volumes in at least some objects at redshifts of 3 . This extends the generality of analogous results for radio galaxies at comparable redshifts (Chambers et al. 1990). As we have emphasized in HLVM, the very existence of the bright, spatially extended regions of $\operatorname{Ly} \alpha$ emission around these QSRs indirectly implies interstellar masses comparable to the baryonic masses of present-day big galaxies $\left(10^{11_{-}}-10^{12}\right.$ $M_{\odot}$ ) to either confine or replenish the Ly $\alpha$ clouds over the lifetime of the radio source (note that these very large masses are even implied by the "resonance-scattering in a hot medium " interpretation of Ly $\alpha$ discussed in $\S 4.7$ above).
While the observed velocity dispersions in the gas are very high $\left(\left[3 \sigma_{\mathrm{los}}\right]^{1 / 2} \approx 10^{3} \mathrm{~km} \mathrm{~s}^{-1}\right)$, they can be understood in terms of gas in free fall into the potential well of a massive galaxy - a process which may be relevant during galaxy formation (see Fall \& Rees 1985; Chevalier \& Lufkin 1990). Alternatively (and more likely in our view), the broad lines in the nebulae may arise in a high-speed galactic-scale outflow. We estimate that the total kinetic energy involved in such an outflow would be $\approx 10^{60}-10^{61}$ ergs, so much or all of the interstellar medium (and possibly a significant fraction of the baryonic mass) of the QSR host galaxy may be lost. It is interesting to consider the role such events might play in the evolution of galaxies and the regulation of the thermal and dynamical properties of the intergalactic medium (see Ikeuchi 1981; Ikeuchi \& Ostriker 1986).

We have noted that the kinematics of the QSR nebulae (broad lines but a lack of organized velocity shears with large amplitude) are qualitatively similar to the kinematics of many "cooling flow nebulae" at low redshifts (see Heckman et al. 1989). However, the QSR nebulae are considerably more kinematically active (typical line widths are several times larger than in the low- $z$ cooling flow nebulae). Thus, our new kinematic data neither strongly support nor conflict with the speculation that high-z QSRs are generically imbedded in cooling flows (e.g., Forbes et al. 1990; Crawford \& Fabian 1989; Fabian 1989). Because the gas motions we observe are supersonic relative to a hot phase with $T \approx 10^{7} \mathrm{~K}$, the quantitative details of the cooling flow models (which assume that the clouds are confined by the static thermal pressure of the cooling flow) may need to be modified when applied to these high-z QSRs.

The images presented in HLVM showed that high-redshift QSRs are broadly similar to radio galaxies with comparable redshifts and radio powers in terms of the sizes and luminosities of the spatially resolved regions of Ly $\alpha$ and UV continuum. The results presented in the present paper demonstrate that the nebulae associated with the two classes are similar kinematically (both have internal velocity dispersions of order $10^{3} \mathrm{~km} \mathrm{~s}^{-1}$ ). Our data on relative line intensities in the high- $z$ QSR nebulae are limited, but are loosely consistent with similar data on radio galaxies ( $\mathrm{Ly} \alpha$ is an order of magnitude [or more] stronger than any other UV lines such as C IV $\lambda 1549$, He II $\lambda 1640$, or C III] 21909 ). It is therefore clear that high- $z$ QSRs and radio galaxies must be closely related phenomena. In particular, our results are consistent with recent speculation that these two AGN classes are related through viewing-angle effects (Barthel 1989). However, we have noted that there may be a systematic kinematic difference between the QSR and radio galaxy nebulae in that the latter often exhibit organized large-scale shears (velocities changing by $\approx 10^{3} \mathrm{~km} \mathrm{~s}^{-1}$ over length scales of many tens of kiloparsecs), while the former do not. If confirmed by analysis of larger samples, this result would pose a challenge for "unification schemes" of the Barthel variety (since a radio-source-driven outflow should be approximately in the sky plane for the radio galaxies and approximately along the line of sight for the QSRs).

We have found suggestive evidence linking the spatially extended Ly $\alpha$ emission to the nuclear $\mathrm{He}$ II $\lambda 1640$ line and to the strong "associated" $z_{\text {abs }} \approx z_{\text {em }}$ systems in high- $z$ QSRs. First (confirming the result of Foltz et al. 1988), the data of BTT show that the nuclear He II line is systematically narrower than other strong nuclear lines (average FWHM $\approx 3000 \mathrm{~km}$ $\mathrm{s}^{-1}$ vs. $6000 \mathrm{~km} \mathrm{~s}^{-1}$, respectively). Second, the equivalent 
width of the nuclear $\mathrm{He}$ II line correlates with the equivalent width of the spatially resolved Ly $\alpha$ emission. We suggest that a significant fraction (half?) of the nuclear $\mathrm{He}$ II emission in high- $z$ QSRs arises in the brightest, centralmost (arcsec-scale) part of the nebula (rather than in the standard broad line region). The gas producing this "narrow" nuclear He II emission will then also produce a nonnegligible portion of the emission in the core of the nuclear Ly $\alpha$ line profile, but will contribute an insignificant amount in other strong nuclear UV lines (e.g., C IV $\lambda 1549, \mathrm{C}$ III] $\lambda 1909$ ). The measured rest equivalent width of the "narrow" nuclear He II line (typically $\approx 5 \AA$ ) implies a covering factor of $\approx 10 \%$ for the gas. This covering factor is consistent with the incidence rate of strong $z_{\text {abs }} \approx z_{\text {em }}$ systems in QSRs (BTT), so that the inner part of the Ly $\alpha$ nebula may correspond to the same gas as the $z_{\mathrm{abs}} \approx z_{\mathrm{em}}$ systems. This is further suggested by the similarity in derived physical conditions in the $\operatorname{Ly} \alpha$ nebulae and the $z_{\text {abs }} \approx z_{\mathrm{em}}$ systems (see HLVM) and by the kinematic similarity of the two phenomena (typical velocities on order of $10^{3} \mathrm{~km} \mathrm{~s}^{-1}$ ).
The authors would like to thank the staff at KPNO for their help in obtaining the data discussed in this paper. We would also like to thank Mike Fall for helpful discussions on infall models, Nick Scoville for badgering us about $\mathrm{Ly} \alpha$ resonance scattering and David Neufeld for helping us understand it, Tim Kallman for kindly providing us with unpublished models of very low metallicity gas photoionized by an AGN continuum, Pat McCarthy and Ken Chambers for useful discussions and for providing us with information about the properties of high- $z$ radio galaxy nebulae, Peter Barthel for discussions about $z_{\text {abs }} \approx z_{\mathrm{em}}$ systems, and Julian Krolik for general discussions of the relevant physics. The work by W. v. B. for this project was performed at IGPP-LLNL under the auspices of the US Department of Energy under contract W-7405-ENG48. Partial support from a NATO research grant and from a visitor grant awarded to $\mathrm{T}$. $\mathrm{H}$. by the Netherlands Organization for Pure Research is gratefully acknowledged.
Anderson, S., Weymann, R., Foltz, C., \& Chaffee, F. 1987, AJ, 94, 278

Barthel, P. 1989, ApJ, 336, 606

Barthel, P., \& Miley, G. 1988, Nature, 333, 319

Barthel, P., Miley, G., Schilizzi, R., \& Lonsdale, C. 1988, A\&AS, 73, 515

Barthel, P., Tytler, D., and Thomson, B. 1990, A\&AS, 82, 339 (BTT)

Begelman, M., \& Cioffi, D. 1989, ApJ, 345, L21

Benvenuti, P., Dopita, M., \& D'Odorico, S. 1980, ApJ, 238, 601

Boroson, T., Persson, S. E., \& Oke, J. B. 1985, ApJ, 293, 120

Boyle, B. 1990, preprint

Carlberg, R., \& Couchman, H. 1989, ApJ, 340, 47

Castor, J., McCray, R., \& Weaver, R. 1975, ApJ, 200, L107

Chambers, K. 1989, Ph.D. thesis, Johns Hopkins University

Chambers, K., Miley, G., \& van Breugel, W. 1990, ApJ, 363, 21

Chevalier, R., \& Lufkin, E. 1990, ApJ, 356, 41

Corbin, M.R. 1989, ApJ, 357, 346

Crawford, C., \& Fabian, A. 1989, MNRAS, 239, 219

Djorgovski, S., Spinrad, H., Pedelty, J., Rudnick, L., \& Stockton, A. 1987a, AJ, 93, 1307

Djorgovski, S., Strauss, M., Perley, R., Spinrad, H., McCarthy, P. 1987b, AJ, 93, 1318

Espey, B., Carswell, R., Bailey, J., Smith, M., \& Ward, M. 1989, ApJ, 342, 666

Fabian, A. 1989, MNRAS, 238, 41P

Fall, S. M., \& Rees, M. 1985, ApJ, 298, 18

Ferland, G., \& Netzer, H. 1983, ApJ, 264, 105

Ferland, G., \& Osterbrock, D. 1986, ApJ, 300, 658

Filippenko, A. 1982, PASP, 94, 715

Foltz, C., Chaffee, F., Weymann, R., \& Anderson, S. 1988, in QSO Absorption Lines: Probing the Universe, ed. J. C. Blades, D. Turnshek, \& C. Norman (Cambridge: Cambridge University Press), 53

Forbes, D., Crawford, C., Fabian, A., \& Johnstone, R. 1990, MNRAS, 244, 680

Garrington, S., \& Conway, R. 1991, preprint

Heckman, T. M., Baum, S., McCarthy, P., \& van Breugel, W. 1989, ApJ, 338, 48

Heckman, T. M., Lehnert, M., van Breugel, W., \& Miley, G. 1991, ApJ, 370, 78 (HVLM)

Hu, E. 1988, in Cooling Flows in Clusters and Galaxies, ed. A. Fabian (Dordrecht: Kluwer), 73

Hu, E., Cowie, L., \& Wang, Z. 1985, ApJS, 59, 447

\section{REFERENCES}

Hu, E., Songaila, A., Cowie, L., \& Stockton, A. 1991, ApJ, 368, 28

Ikeuchi, S. 1981, PASJ, 33, 211

Ikeuchi, S., \& Ostriker, J. 1986, ApJ, 301, 522

Isobe, T., Feigelson, E., \& Nelson, P. 1986, ApJ, 306, 490

Krolik, J., McKee, C., \& Tarter, C. 1981, ApJ, 249, 442

McCarthy, P. 1988, Ph.D thesis, University of California, Berkeley

McCarthy, P., Spinrad, H., Djorgovski, S., Strauss, M., \& van Breugel, W. 1987, ApJ, 319, L39

McCarthy, P., Spinrad, H., van Breugel, W., Liebert, J., Dickinson, M., Djorgovski, S., \& Eisenhardt, P. 1990, ApJ, 365, 487

Miley, G. K. 1971, MNRAS, 152, 477 1980, ARA\&A, 18, 165

1987, in IAU Symp. 124, Observational Cosmology, ed. A. Hewitt, G. Burbidge, \& L. Fang (Dordrecht: Reidel), 267

Neff, S., \& Hutchings, J. 1990, AJ, 100, 1451

Neufeld, D. 1990, ApJ, 350, 216

Neufeld, D., \& McKee, C. 1988, ApJ, 331, L87

Neugebauer, G., et al. 1980, ApJ, 238, 502

Norman, C., \& Miley, G. K. 1984, A\&A, 141, 85

Osterbrock, D., and Mathews, W. 1986, ARA\&A, 24, 171

Rees, M. 1988, MNRAS, 231,91P

Scarrott, M., Rolph, C., \& Tadhunter, C. 1990, MNRAS, 243, 5P

Shull, S. M., \& McKee, C. 1979, ApJ, 227, 122

Spinrad, H., \& Djorgovski, S. 1984, ApJ, 280, L9

Stasinska, G. 1990, A\&AS, 83, 501

- 1984, A\&AS, 55, 15

Steidel, C., Sargent, W., \& Dickinson, M. 1991, preprint

Stewart, G., Canizares, C., Fabian, A., \& Nulsen, P. 1984, ApJ, 278, 536

Stockton, A., \& MacKenty, J. 1987, ApJ, 316, 584

Urbaniak, J., \& Wolfe, A. 1981, ApJ, 244, 406

van Breugel, W., Heckman, T., Miley, G., Butcher, H., Bridle, A. 1985, ApJ, 290,496

van Breugel, W., Heckman, T., Miley, G., \& Filippenko, A. 1986, ApJ, 311, 58

Weymann, R., Williams, R., Peterson, B., Turnshek, D. 1979, ApJ, 234, 33

Whittle, M., Pedlar, A., Meurs, E., Unger, S., Axon, D., \& Ward, M. 1988, ApJ, 326,125

Williams, R., Strittmatter, P., Carswell, R., \& Craine, E. 1975, ApJ, 202, 296 